\title{
Real-Time, Wearable, Biomechanical Movement Capture of Both Humans and Robots with Metal-Free Electrodes
}

\author{
Priya Rathi, ${ }^{\dagger}$ Mihir Kumar Jha, ${ }^{\dagger}$ Kenji Hata, ${ }^{\dagger}$ and Chandramouli Subramaniam* ${ }^{*} \dagger$ (]) \\ ${ }^{\dagger}$ Department of Chemistry, Indian Institute of Technology Bombay, Powai, Mumbai 400076, Maharashtra, India \\ ${ }^{\ddagger}$ Nanotube Research Center, National Institute of Advanced Industrial Science and Technology (AIST), Tsukuba 305-8565, Japan
}

\author{
Supporting Information
}

\begin{abstract}
We demonstrate an all-carbon-based, flexible, conformal movement-capturing device capable of precisely monitoring biomechanical movements of both humans and robots. Mechanically robust, metal-free electrodes form a unique component of the device responsible for qualitatively and quantitatively transducing biomechanical movements without any signal artifacts. Importantly, the device withstands and operates in a wide dynamic range for both stretching (25\% strain) and bending $\left(140^{\circ}\right)$ actions with minimal cycling hysteresis (2.0), high repeatability (>100 cycles), low creep,

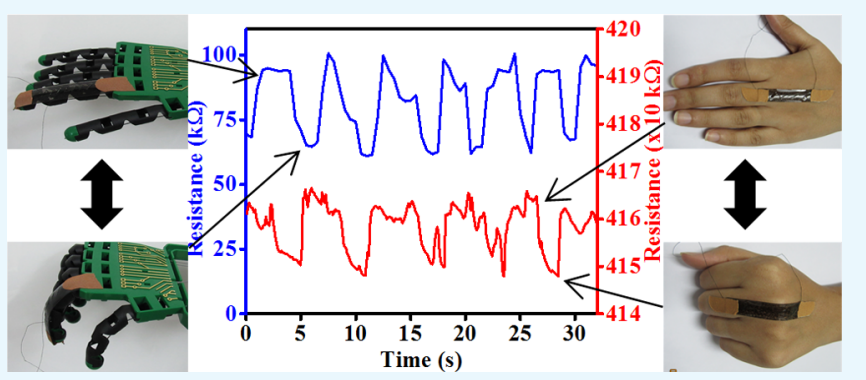
and humidity-independent rapid response $(\sim 200 \mathrm{~ms})$.

Furthermore, the device qualitatively distinguishes movements such as bending of finger, knuckle, and wrist and also provides quantitative information on the extent of such movements. We establish that single-wall carbon nanotubes (CNTs) embedded in ultralow concentration $(0.016 \mathrm{wt} \%)$ within an elastomeric matrix undergo three-dimensional conformational changes during biomechanical movements that are subsequently transduced as signals. In addition, such CNT-elastomer strips exhibit enhanced stretchability $(>100 \%)$ and elasticity $(\sim 77 \%)$ in comparison to those of pure elastomers, leading to a wider dynamic working range of the device. Furthermore, seamless integration of a versatile gesture tracker on ubiquitous platforms, such as human skin, kinesiologic tapes, gloves, and robotic arms, is achieved, thereby catering to applications ranging from healthcare monitoring and physiotherapy to robotics and wearable technologies.
\end{abstract}

\section{INTRODUCTION}

Kinesiologic monitoring of human motion has been garnering increasing attention in diverse areas, such as wearable technologies, personalized point-of-care health monitoring systems, military, and robotics. ${ }^{1-3}$ Furthermore, several physiotherapeutic restorative procedures such as stroke rehabilitation and prosthetic compatibility demand precise and point-of-care kinesiologic monitoring and tracking of various gestures. There has also been a paradigm shift in computing, toward technologies employing gesture tracking as input. Such gestures are often composed of multiaxial movements, such as bending, flexing, and twisting of various limbs. A device capable of achieving such kinesiologic movement capture has to exhibit a wide dynamic working range, high sensitivity to movements, ability to distinguish different types of movements, and quantify the extent of movement in addition to biocompatibility, minimal invasiveness, mechanical robustness, and ease of use.

Toward this end, technologies aimed at capturing human motion are predominantly based on either micro-electromechanical systems (MEMSs) ${ }^{4-6}$ or electrically conducting polymer composites (ECPCs) $)^{7-10}$ or optical motion-capture techniques. ${ }^{11}$ Although MEMS technologies rely on engineering novel architectures with $\mathrm{Si}$ as a platform, ECPCs pertain to a class of devices fabricated on stretchable platforms. The latter class also includes elastomeric composites with conductive fillers, such as carbon nanotubes (CNTs) fabricated on a variety of substrates, resulting in piezoresistive ECPCs capable of capturing human motion. ${ }^{12-17}$ Such polymer-based platforms exhibit high degree of mechanical robustness but suffer from poor sensitivity toward precisely detecting mechanical movement. Furthermore, the performance of such devices exhibits a strong dependence on their ambience, such as relative humidity (RH) and temperature, resulting in ambiguity in the interpretation of the signals. In contrast, MEMS-based platforms are very sensitive and precise and are not affected by environmental conditions but are limited by their dynamic working range and challenges in direct interfacing with the epidermal layer. Importantly, designing electrical contacts to both these classes of devices (MEMS and ECPCs) has been a persistent challenge due to the ineffectiveness and failure of the contacts when exposed to physical actions, such as stretching and bending. Optical motion-capture techniques provide realtime precision but require dedicated environments and a host of cumbersome facilities and thereby are not currently adaptable for personalized applications. Thus, a singular,

Received: April 21, 2017

Accepted: July 18, 2017

Published: August 2, 2017 


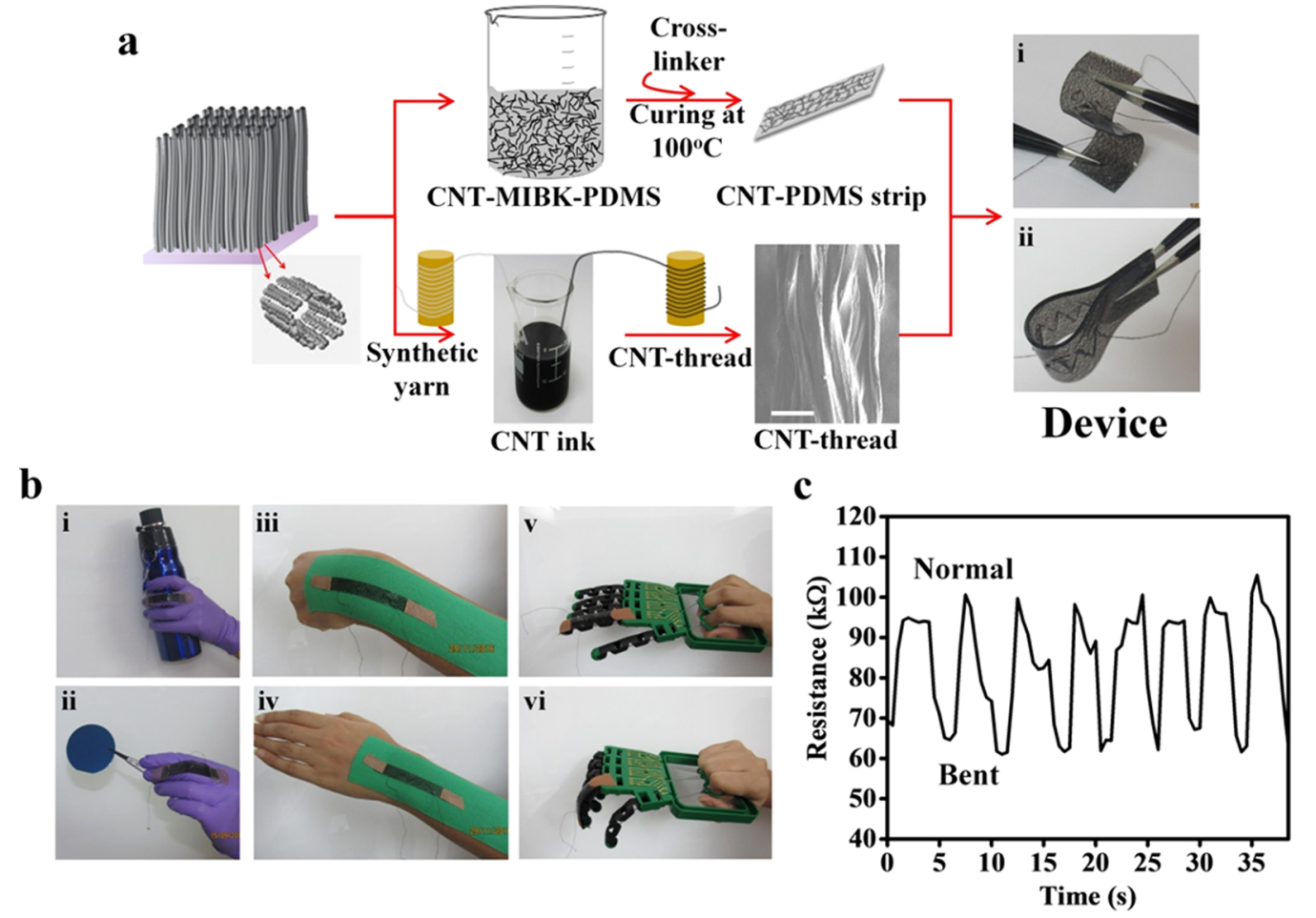

Figure 1. Fabrication and versatile demonstration of the device. (a) Key steps in device fabrication, culminating in a flexible, mechanically robust device. The scanning electron microscopy (SEM) of the CNT thread (scale bar $100 \mu \mathrm{m}$ ) along with photographs of the device in (i) S-shaped and (ii) U-shaped bent configurations. (b) Demonstration of integrability of the device on ubiquitous platforms, such as on (i, ii) NBR gloves, (iii, iv) kinesiologic tapes, and (v, vi) artificial robotic arm without inhibiting any mechanical action, as illustrated by (i) holding a bottle and (ii) using a forceps. (c) Real-time response generation from gesture tracker during physical movement of the robotic arm. Photograph courtesy of C.S. Copyright 2016.

biocompatible device exhibiting conformal attachment to the epidermal layer of skin and possessing the ability of detecting and precisely quantifying the movement of the associated limbs without compromising on the sensitivity and dynamic range is highly desirable.

Addressing this demand, we report a kinesiologic, bodily movement-capturing device composed of a CNT-elastomeric strip that is capable of precisely tracking multidirectional and multiaxial movements associated with human limbs over a wide dynamic range (150\%), exhibits instantaneous response ( 200 $\mathrm{ms}$ ), and has high reliability and reproducibility with low creep and minimal hysteresis (2.0 for more than 100 cycles). We demonstrate the real-time qualitative and quantitative tracking of hand gestures that employ a combination of modes, such as stretching and bending. Furthermore, the device not only distinguishes different types of actions but also provides signals that can be quantitatively assigned to the extent of such actions. Thereby, the device generates signals that can be used to differentiate actions that employ the same set of musculoskeletal joints, such as holding a pen or a bottle or clenching a fist. We show that the CNTs, oriented as three-dimensional (3D) random networks at a near-percolation threshold concentration in the poly(dimethylsiloxane) (PDMS) matrix, undergo distinct conformational changes under a mechanical stress. Such conformational changes in the CNTs are transduced using CNT-immobilized yarns embedded in elastomers and are used as contacts for signal read-out, thereby overcoming the challenges associated with electrical contacting of such elastomeric devices. Finally, we demonstrate integration of the device onto a variety of platforms, such as human skin, kinesiologic tapes, robotic arms, and nitrile butadiene rubber (NBR) gloves.

\section{RESULTS}

Realization of a kinesiologic, movement-capture device involves symbiotic integration of various critical processes and materials, as illustrated in Figure 1a. PDMS is chosen as the device platform due to its biocompatibility, mechanical robustness, and chemical inertness. CNTs with high-aspect ratios $\left(>10^{5}\right)$ and high surface areas $\left(\sim 1000 \mathrm{~m}^{2} / \mathrm{g}\right)$ were utilized as a functional component of the device. Uniform dispersion of the functional component (CNTs) in the viscoelastic matrix (PDMS) is critical for achieving reliable and reproducible performance of device. Considering the viscous nature of monomeric PDMS, direct dispersion of CNTs proved to be a challenge. This was overcome by employing methyl isobutyl ketone (MIBK) as an intermediary medium for dispersing CNTs, thereby ensuring a solvent-mediated dispersion of CNTs into monomeric PDMS. The choice of MIBK as the intermediary medium originates from its similarity in the solubility parameter to $\mathrm{CNTs},{ }^{19-21}$ which ensured their minimal structural damage and maximum dispersion in the PDMS matrix. Significantly, such an approach also resulted in a substantially lower percolation threshold of CNTs (0.016 wt $\%$ ) compared to that in previous reports. ${ }^{22}$ These low loading levels correspond to the weight fraction of CNTs in the PDMS matrix and resulted in an electrical conductivity of $\sim 3 \times 10^{-6}$ $\mathrm{S} / \mathrm{cm}$. Subsequent cross-linking and curing resulted in the formation of free-standing, flexible, and mechanically robust CNT-PDMS strips (Figure 1a). The uniform dispersion of 


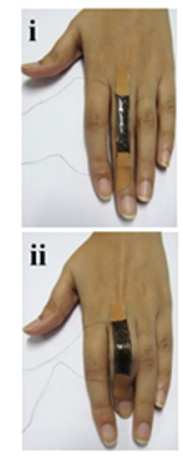

b

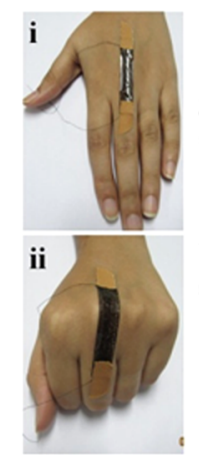

c

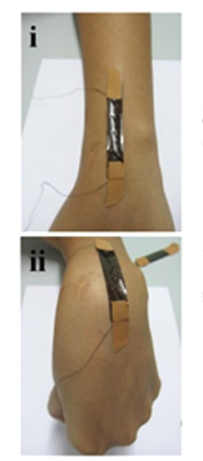

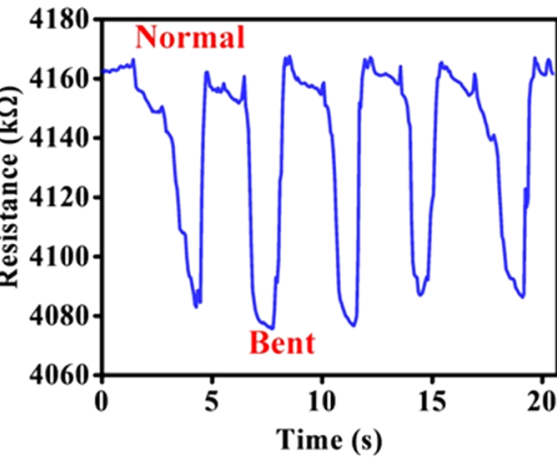

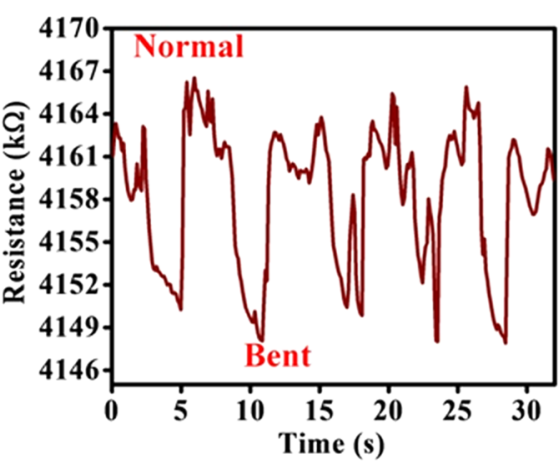

d

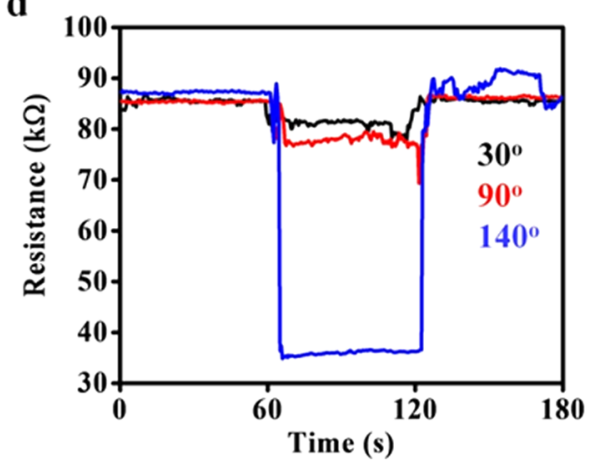

e

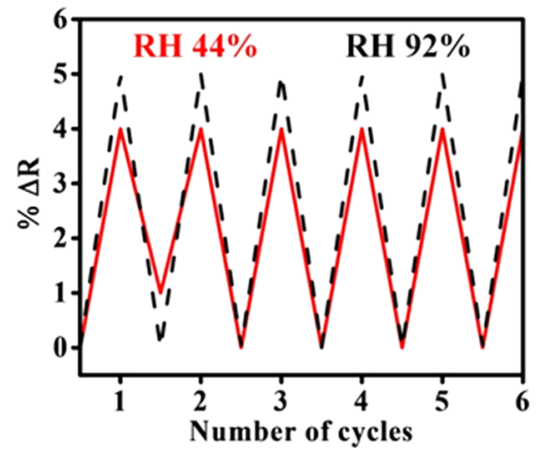

f
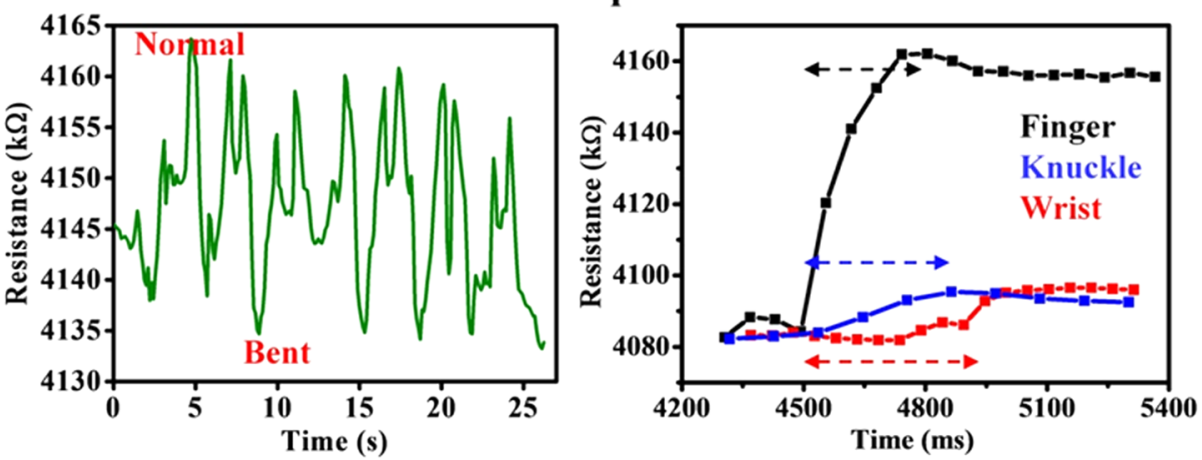

Figure 2. Real-time signal generation from gesture tracker corresponding to bending of IP (a), MP (b), and CM (c) joints. Photos (i) and (ii) correspond to the normal and bent configurations of the respective joints. (d) Variations in the signal generated by bending the same joint to differing extents. (e) Performance of the device under different ambient humidity conditions (44 and 92\%). (f) Evolution of signals from the three joints described in (a)-(c). Photograph courtesy of M.K.J. Copyright 2016.

CNTs in the final device is reflected as a systematic decrease in transmittance of the CNT-PDMS strips because CNTs are known to be perfect blackbody absorbers in the visible region of the electromagnetic spectrum ${ }^{23}$ (see the Figure S1, Supporting Information). Finally, incorporation of a transducer involved interlacing through the CNT-PDMS strips, resulting in the final device.

Importantly, the transducer also serves as flexible and mechanically robust electrodes during measurements and has been easily integrated onto a variety of substrates, in contrast to other reports involving copper wire and silver paste contacts ${ }^{24}$ and gold electrodes. ${ }^{25}$ Thereby, the signal read-out is facilitated without any motion-induced artifacts from the electrodes. In addition, the presence of any metallic component often leads to false read-outs and failure of the device due to resistive changes produced in the metallic films and contacts, during the mechanical movements. In fact, this is the primary mechanism operating in metal-film-based resistive strain gauges that exhibit a limited dynamic working range. In sharp contrast, we demonstrate the working of our device without any metallic component and thereby overcome all of these shortcomings to constituting an important advancement toward wearable components.

The final device thus fabricated (Figure 1a) is a synergistic combination of the mechanical robustness of the elastomeric matrix and the conductive functionality of CNTs. Furthermore, the device is composed of (a) a $3 \mathrm{D}$ network of randomly oriented, interconnected CNTs that are embedded in PDMS and (b) uniformly coated CNTs immobilized on synthetic yarns. The CNTs form a uniform, conformal coating on the multiply cellulose thread, as indicated in the SEM image (see Figures 1a and S2). Such a CNT-based coating results in constriction of the thread diameter, owing to strong $\pi-\pi$-based van der Waals interactions between the CNTs, resulting in the final diameter of the CNT thread to be $\sim 200 \mu \mathrm{m}$ ( $40 \%$ reduction in diameter). Significantly, the CNT thread is also electrically conductive, as demonstrated in Figure S3, Supporting Information. 
Owing to the flexible and elastomeric nature of the constituents, the device could be twisted and contorted into a variety of shapes, such as an S-shape involving combination of two opposing $180^{\circ}$ bends (Figure 1a-i) and a U-shape involving a $360^{\circ}$ bend (Figure 1a-ii), without any loss of device integrity. Furthermore, a variety of physical actions, such as holding a bottle (Figure $1 \mathrm{~b}-\mathrm{i}$ ) and using a forceps (Figure $1 \mathrm{~b}-\mathrm{ii}$ ), can be carried out without any hindrance or restriction from the device. Importantly, all of these aspects apply uniformly to every part of the device unlike previous reports ${ }^{12}$ wherein the contact for signal acquisition has to be preserved in a rigid environment to prevent contact-induced signal distortions. All of the above-mentioned features are corroborative in nature and point to mechanical integrity of the device during various modes of movement exhibited by humans, machines, and their interfaces.

Importantly, the device conformally attaches to a variety of soft and hard surfaces, such as the epidermal layer of human skin, NBR gloves (Figure 1b-i,ii), kinesiologic tapes (Figure 1biii,iv), plastic-based robotic arms (Figure $1 \mathrm{~b}-\mathrm{v}, \mathrm{vi}$ ), glass and silicon surfaces, an aspect critical for versatile usage ranging from humans to robots. Although van der Waals in nature, a medical adhesive assisted the attachment and prevented any slippage during the measurements. The device remained adherent and retained its configuration and functionality for over a month on all of these surfaces.

The electrical resistance across the device was monitored using the CNT thread as a transducer during repeated movements at the desired locations. Systematic measurements were carried out by bending the robotic arm and monitoring the changes in resistance produced during the movements. A highly reproducible and reversible change in the resistance (40 $\mathrm{k} \Omega$ ) across the device, pertaining to the normal (Figure 1b-v) and bent states of the robotic arm (Figure $1 \mathrm{~b}$-vi), was observed during such measurements. In addition, the response from the device reverts back to the original state upon relaxation of the robotic arm, indicating minimal fatigue and hysteresis (Figure 1c). Detecting humanlike movements in real time, generated artificially on differing platforms, signifies an important step toward the development of robots, artificial muscles, and robotassisted surgeries in healthcare.

Extending this for detecting human motion, the device could be conformally attached at specific locations on a human hand, such as the proximal interphalangeal (IP) joint, metacarpophalangeal (MP) joint, and carpometacarpal (CM) joint. These proximal joints are predominantly involved in dexterity and gesturing and thus are of interest for the proposed applications. The angle of bending is defined as the angle described by the joint in moving from its normal configuration (Figure 2a-i) to the bent configuration (Figure 2a-ii). The tracking signal constituted a resistance change and is monitored both during the course of movements and in the final positions. Specifically, a smooth, continuous decrease in resistance by $\sim 80 \mathrm{k} \Omega$ is observed during the bending of the IP joint across a maximum permissible angle of $140^{\circ}$ (Figure $2 \mathrm{a}$ ). Consequent relaxation to the normal configuration resulted in restoration to the original value $(4160 \mathrm{k} \Omega$ ). Repeated cycling of the device (over 100 cycles) through the action of the IP joint produces precise, highly reproducible, and consistent signals. Such uninterrupted, in situ motion capturing demonstrated with our device indicates its substantial advantage over conventional MEMS platforms that are capable of detecting only the initial and final positions and not the intermediary states.
Extending the versatility of the gesture tracking device, it is observed that action of other joints, such as MP (Figure 2b) and $\mathrm{CM}$ (Figure 2c), on the same hand produces distinctly different signals that are both indicative of the site of bending and proportional to the angle of bending. Thus, distinctly different signals are obtained across the three joints investigated (IP, MP, and CM joints), in spite of their proximity to each other. Common to all of these signals is the fact that the resistance decreases upon bending of the joint and is restored to its initial value upon relaxation of the joint. The absence of any hysteresis during repeated measurements indicates no mechanical fatigue of the device and augurs well for its longterm usability. Importantly, the magnitude of resistance change is observed to be strongly associated with the extent of mechanical movement occurring at a particular joint. The three joints tested here bend to different extents (maximum bending of 140,90 , and $80^{\circ}$ for IP, MP, and CM joints, respectively; Figure S4, Supporting Information) and correspond to the bending of finger, knuckle, and wrist. Accordingly, changes in resistance pertaining to these movements are $80 \mathrm{k} \Omega$ (IP), 20 $\mathrm{k} \Omega(\mathrm{MP})$, and $25 \mathrm{k} \Omega(\mathrm{CM})$, respectively, and are thus proportional to the extent of biomechanical movement. Furthermore, the device exhibits the ability to distinguish between differing extents of movement occurring across a given joint. This is illustrated with experiments performed by bending the IP joint to differing extents ranging from 30 to $140^{\circ}$ (Figure $2 \mathrm{~d})$. It is to be noted that these angles correspond to gestures such as holding a bottle $\left(30^{\circ}\right.$ bending), using a forceps $\left(90^{\circ}\right.$ bending), and a clenched fist ( $140^{\circ}$ bending). Distinctly different changes in resistance from the device (signals) located at the IP joint are observed corresponding to each of the gestures made, with the magnitude of the signal being proportional to the extent of bending. Thus, gesturing of a clenched fist produces a higher change in the resistance (55 $\mathrm{k} \Omega$ ) as compared to that from holding a bottle $(10 \mathrm{k} \Omega)$ or using a forceps $(5 \mathrm{k} \Omega)$, correlating to the associated bending angles. Thus, a unique ability to qualitatively distinguish between the type of action and simultaneously quantify the extent of action at the site of various musculoskeletal joints is demonstrated.

Additional advantage of the device lies in its robustness to its immediate ambience, specifically humidity. Although the absolute value of resistance is inherently dependent on the ambient humidity, the response of the device to biomechanical movements is observed to be invariant to the humidity levels ranging from $\mathrm{RH} 40$ to $90 \%$ (Figure 2e). The CNT thread is able to reliably transduce the signals from the CNTs embedded in PDMS, thereby retaining the device performance without the requirement of any additional packaging. Thus, the tracking signal (resistance change) is independent of the ambient humidity, exhibiting distinct advantages over that from previous reports that either exhibited a humidity-dependent response or required additional packaging to shield it from ambience. ${ }^{12}$ Such a humidity-invariant response is particularly important for applications related to direct interfacing with skin, which experiences profuse variations in humidity. ${ }^{26}$

Real-time movement capture also demands the generation of instantaneous response across a wide dynamic working range. To evaluate this aspect, the evolution of signal during both the bending of the biomechanical joints (IP, MP, and CM) and their subsequent relaxation to the normal position was analyzed (Figure 2f). The response time of the device is defined as the time delay between the start of the movement and the 
a

c

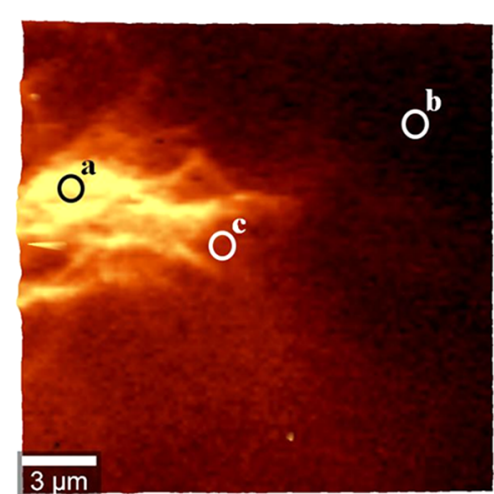

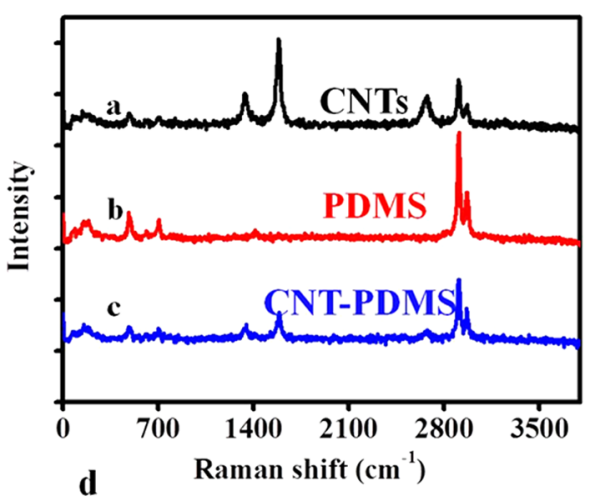

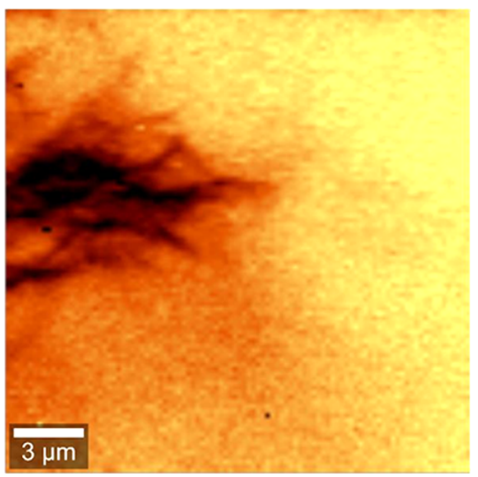

Figure 3. Microscopic and spectroscopic 3D structure. (a) Four-dimensional X-ray microscopic image of the 3D volume element of the device. (b) Raman spectra corresponding to different positions on the image. Micro-Raman spectral mapping of CNT-PDMS based on (c) CNTs $\left(1580 \mathrm{~cm}^{-1}\right)$ and (d) PDMS $\left(2940 \mathrm{~cm}^{-1}\right)$ indicative of regions occupied by the (a) filler (yellow), (b) matrix (black), and (c) homogenous filler-matrix interface (red).

stabilization of the signal. It is observed that the response time of the device is independent of the initial and final states during the action and is determined by the extent of action occurring at the joint. Thus, the bending of finger involving the largest arc length $(9.7 \mathrm{~cm})$ produces the quickest time for response and restoration $(\sim 300 \mathrm{~ms})$, whereas the bending of wrist involving the smallest arc length $(5.5 \mathrm{~cm})$ exhibits the slowest response and restorative time $(\sim 550 \mathrm{~ms})$ (Figure $2 \mathrm{f})$. The action of knuckle also follows this trend with an arc length of $6.3 \mathrm{~cm}$ and a response time of $\sim 400 \mathrm{~ms}$. Significantly, experiments carried out with robotic arms, capable of faster movements, generate a quicker response from the device ( $200 \mathrm{~ms})$ (Figure 1c). It is to be noted that all of the measurements were carried out in time steps of $50 \mathrm{~ms}$. The response time ranging from 300 to $550 \mathrm{~ms}$ is of similar order, as observed in a previous report. ${ }^{27}$ Although the response of our device is 1 order of magnitude slower than the best reported value, ${ }^{12}$ the ease of fabrication, absence of metallic contacts as electrodes, and extensive packaging are the significant advantages for commercial realization of the technology.

The various capabilities of the device demonstrated here can be directly correlated to the structural organization of CNTs in the PDMS matrix and the resulting architecture of the device. A variety of approaches were adopted to understand the nature of distribution and networking of CNTs in the PDMS matrix. First, a $3 \mathrm{D}$ volume element $\left(15 \times 15 \times 15 \mu \mathrm{m}^{3}\right)$ of the device was reconstructed from two-dimensional (2D) images generated on the basis of contrast difference between iodine-doped $\mathrm{CNTs}^{28}$ (see Figure S5, Supporting Information) and PDMS, through four-dimensional X-ray microscopy (Figure 3a). Thus, the darker areas of the volume element correspond to the presence of iodine-doped CNTs and the lighter areas correspond to the carbon framework of the elastomeric matrix. As shown (Figure 3a), the dark and light areas of the reconstructed volume element show a $3 \mathrm{D}$, continuous, interpenetrating structure, indicative of homogenous distribution of CNTs in the PDMS matrix. Furthermore, the relatively equal proportion of the light and dark areas indicates the absence of any significant phase separation or domain formation. The high-aspect ratio $\left(>10^{5}\right)$ and specific surface area $\left(\sim 1000 \mathrm{~m}^{2} / \mathrm{g}\right)$ of the CNTs are critical to achieve such high volume coverage by CNTs, in spite of their low weight fraction (0.016 wt \% CNTs).

Second, more detailed aspects of the CNT distribution are investigated through confocal micro-Raman spectroscopy that relies on the distinctly different vibrational signatures of the CNTs and PDMS. The characteristic peaks including radial breathing mode, D-band, and G-band are observed for CNTs at 182,1344 , and $1580 \mathrm{~cm}^{-1}$, respectively. Similarly, the peaks centered at 2940,717 , and $500 \mathrm{~cm}^{-1}$ are attributed to $\mathrm{C}-\mathrm{H}$ stretching, $\mathrm{Si}-\mathrm{C}$ symmetric stretching, and $\mathrm{Si}-\mathrm{O}-\mathrm{Si}$ stretching modes, respectively (Figure $3 \mathrm{~b}$ ). Thus, confocal spectroscopic mapping based on the peaks centered at $1580 \mathrm{~cm}^{-1}$ (G-band, Figure 3c) and $2940 \mathrm{~cm}^{-1}(\mathrm{C}-\mathrm{H}$ stretching, Figure 3d) are carried out. The randomly distributed nature of the CNT network mimicking polymeric chains is evident from such spectral mapping. Furthermore, the CNTs are seen to branch out from each other while remaining connected at discrete parts in a fractal-like geometry. In fact, the spatial distribution of the CNTs in the PDMS matrix resembles dendritic structures with a dense core and arms spreading out in all three directions (see Figure S6, Supporting Information). In 
a

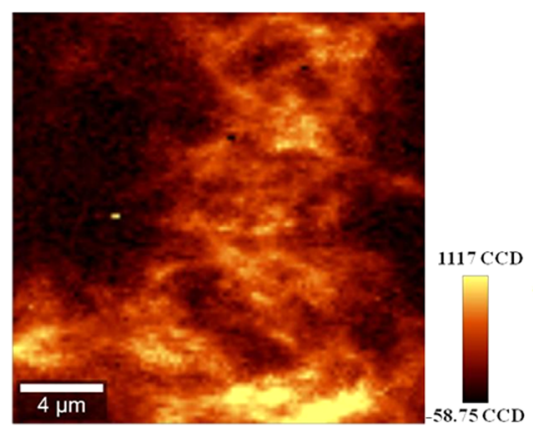

b

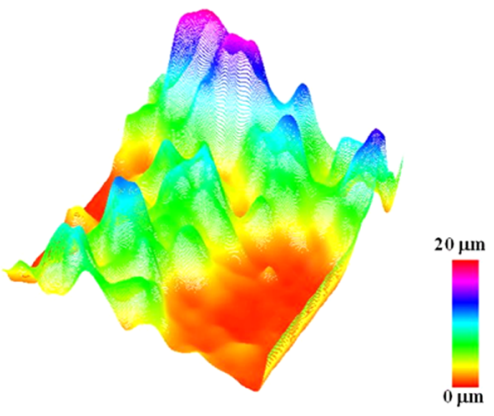

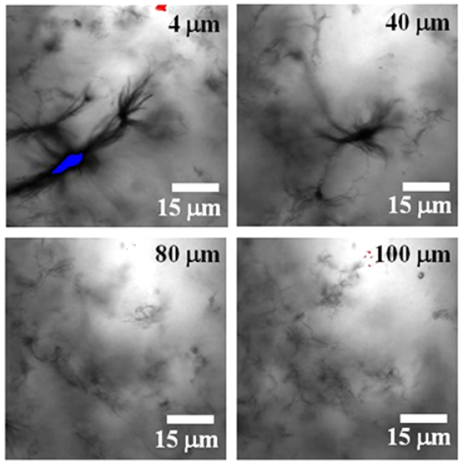

d

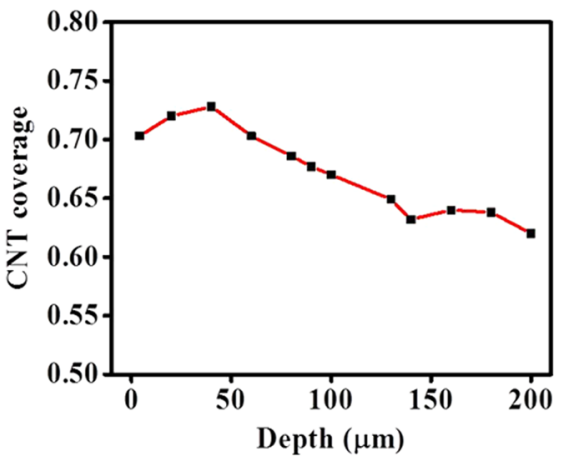

Figure 4. Quantitative and spectral mapping of CNTs. (a) Overlapped spectral confocal images based on the G-band $\left(1580 \mathrm{~cm}^{-1}\right) \mathrm{collected} \mathrm{across} \mathrm{a}$ depth of $10 \mu \mathrm{m}$ with a $z$-step size of $1 \mu \mathrm{m}$. (b) Three-dimensional representation of confocal spectral maps with the $z$ axis color bar pertaining to various depths of the device. (c) Confocal laser scanning images of the device corresponding to $0.016 \mathrm{wt} \%$ of CNTs, recorded at sample depths of (i) $4 \mu \mathrm{m}$, (ii) $40 \mu \mathrm{m}$, (iii) $80 \mu \mathrm{m}$, and (iv) $100 \mu \mathrm{m}$. (d) CNT coverage at different depths of the device.

addition, Raman spectra collected at different points of the spectral map indicate a large area that is primarily composed of the interface between CNTs and PDMS (Figure 3c). This further confirms the fractal-like distribution that provides it a large interface area with PDMS. Thereby, seamless integration into each other, an aspect critical for the low fatigue and mechanical reinforcement, is observed in the device.

Having spectroscopically and microscopically established the 2D network structure of CNTs, the analysis was extended to confocally image a $3 \mathrm{D}$ volume element of the device $(20 \times 20$ $\left.\times 10 \mu \mathrm{m}^{3}\right)$. Such an analysis is critical for correlating the observed properties to the $3 \mathrm{D}$ microscopic structure of the device. Confocal 2D spectral maps taken at different depths of the sample (see Figure S7, Supporting Information) were overlapped to generate a $3 \mathrm{D}$ distribution profile of CNTs in the device (Figure 4a). This reveals the continuous nature and fractal geometry of the CNTs extending across all three dimensions of the device. The spectral maps taken at different depths $(1 \mu \mathrm{m}$ apart $)$ of the sample are combined to illustrate the nature of such continuous, fractal-like geometric distribution of CNTs. Represented three dimensionally (Figure 4b), it reiterates the microstructure distribution of CNTs that traverses all such confocal layers in a continuous fashion and thereby electrically interconnects all of these layers.

Finally, quantification of the CNT coverage is carried out through confocal transmission microscopy (Figure 4c). The images collected at different depths are analyzed (ImageJFlaxLac_2,5) to form 2D binary black and white images. Because CNTs are known to be blackbody absorbers, ${ }^{23}$ any black region corresponds to CNTs and optically transparent region corresponds to elastomeric matrix. Thereby, the CNT coverage in each confocal layer is quantified by estimating the proportion of black area to the total area sampled (Figure $4 \mathrm{~d}$ ). The CNT volume coverage is found to be $\sim 65 \%$ irrespective of the sampling depth (see Table S1 and Figure S8a, Supporting Information). Besides indicating the uniformity of CNT dispersion, this also indicates the high volume fraction coverage of CNTs $(\sim 65 \%)$ that has been achieved at low loading levels (0.016 wt \%). Reinforcing these observations, the crosssectional SEM images indicate a homogenous, continuous, networked structure of CNTs when dispersed in PDMS (Figure S8b, Supporting Information). Importantly, there is no phase separation or segregation of the CNTs and PDMS in the strip, an aspect critical for the effective functioning of the device. Thus, the use of CNTs with high-aspect ratios $\left(>10^{5}\right)$ is absolutely essential because the percolation threshold of the filler varies inversely with its aspect ratio, that is, spherical conductive fillers, such as activated carbon and carbon black, would be required in much higher concentrations to establish continuous conductive pathways in comparison with fibrous structures, such as nanotubes (see Figure S6, Supporting Information).

Combined analyses of all of these approaches reinforce the importance of high-aspect ratio CNTs and the importance of the methodology adopted for device fabrication. Thus, even though the CNTs are employed at an ultralow, near-percolation threshold concentration ( 0.016 wt \%), the effectiveness of the dispersion ensures their uniform and three dimensionally continuous, interlinked networking through the elastomeric matrix, thereby providing an exceptionally high volume coverage $(\sim 65 \%)$.

Such homogeneous blending of CNTs and PDMS is reflected in the exceptional enhancement of the mechanical properties of the device compared to that of pristine PDMS. 
a

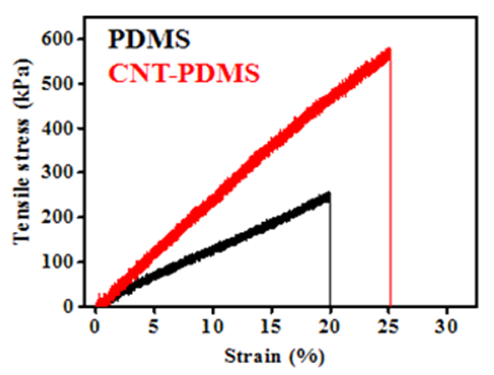

f

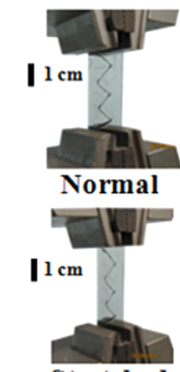

b
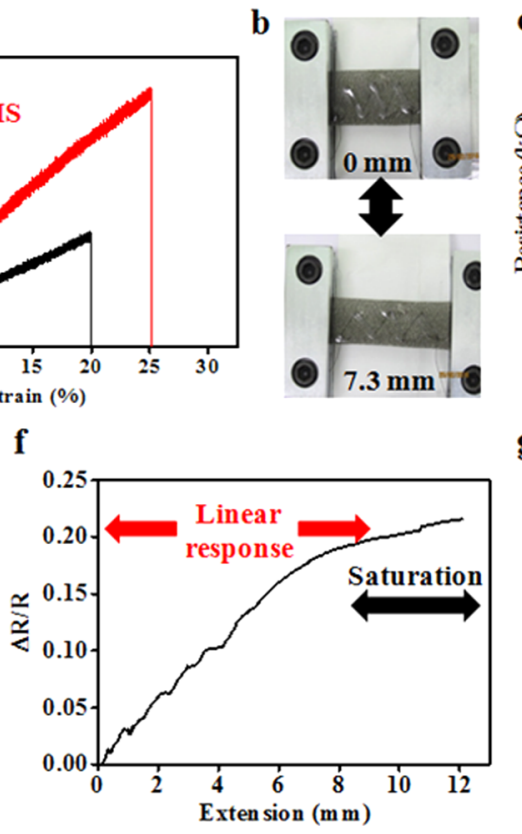

c

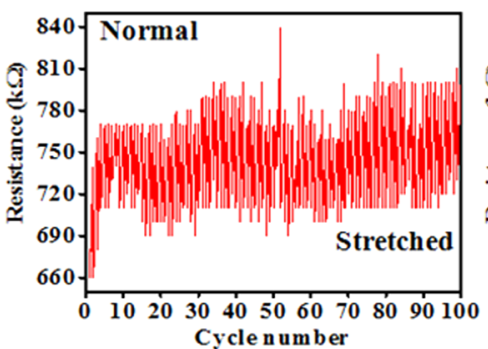

g

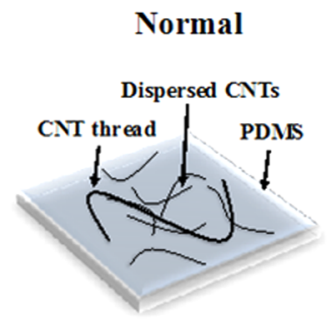

d

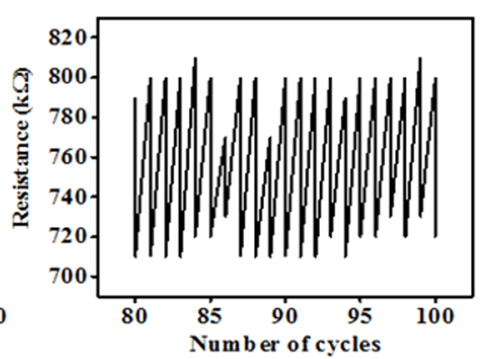

Stretched

Figure 5. Underlying working mechanism of the device. (a) Tensile stress vs strain plots for pure PDMS (black) and CNT-PDMS (red). (b) Images of the device in normal $(0 \mathrm{~mm})$ and stretched $(7.3 \mathrm{~mm})$ configurations during its repeated, cyclic tensile testing. (c) Signal generated by device corresponding to the normal and stretched states, as indicated in (b) upon repeated cyclic testing. (d) Signal response of device for 10 such cycles. (e) Configurations of the device corresponding to the normal and stretched states during in situ electromechanical measurement. (f) In situ electromechanical measurement showing the change in resistance with extension of the device. $(\mathrm{g})$ Schematic representation of the normal and stretched states of the device. The compressive stress (blue arrows) generated due to the tensile stress (green arrows) results in increased contacts of the dispersed CNTs with the CNT thread, leading to reduced resistance.

Tensile testing of the device indicates a substantial increase in both the Young modulus and the ultimate tensile strength (UTS) of the device when compared to those of pristine PDMS (Figures 5a and S9a). Specifically, the Young modulus is enhanced by $77 \%$ from $1.3 \mathrm{MPa}$ (pure PDMS) to $2.3 \mathrm{MPa}$ (device) with the UTS increased by over $100 \%$. Concurrently, the yield strain of the device is observed to increase by $25 \%$. Addition of CNTs produces a reinforcement effect on the PDMS matrix, which can be attributed to two synergistic effects: (a) extended polymerization of the monomeric dimethylsiloxane through free-radical stabilization by $\mathrm{CNTs}^{29,30}$ and (b) enhanced dispersion of CNTs and PDMS due to their similar solubility parameters. ${ }^{17-19}$ Thus, the cumulative effect of CNT addition results in (a) increased stretchability (high UTS and high yield point), leading to a wider dynamic working range of the device, and (b) enhanced elastic reversibility (high Young's modulus), resulting in its low creep, low fatigue, and higher repeatability and reusability. Considering that such significant enhancements are generated due to addition of ultralow concentration $(0.016 \mathrm{wt} \%$ and 65 vol \%) of CNTs, a strong correlation between the 3D, structurally interconnected pathways of CNTs in the PDMS matrix and the mechanical reinforcement is conclusively established. It is to be noted that similar strain sensors employing CNTs work at significantly higher filler loading that could lead to self-aggregation-induced degradation of mechanical properties. Therefore, the high-aspect ratio $\left(>10^{5}\right)$ of CNTs and their seamless integration into PDMS operate symbiotically to mechanically reinforce the device.

Reproducible operation and reliable signal generation form an important aspect of such biomechanical motion-capture devices. Quantification of these aspects was carried out by repeated cyclic tensile testing of the device using a custom-built jig (see Section 4 for details) and simultaneously monitoring its electrical response (Figure $5 b$ ). Unlike real-time biomechanical tracking, such testing enabled quantifiable and reproducible loading-unloading tensile stress-strain cycles to be carried out on the device to gauge its performance (Figure 5c). The device exhibits a highly uniform response over 100 loading-unloading cycles with a standard deviation (SD) of 2.0 (Figure $5 \mathrm{~d}$ ). We also note that a device fabricated with a shorter CNT thread length (transducing element) provided a low SD of 0.06 with a relative $\mathrm{SD}$ of $0.47 \%$ (see Figure S9b,c, Supporting Information). This confirms the absence of any mechanical fatigue or failure due to repeated usage and is in excellent agreement with the enhanced elasticity and UTS values reported here.

Critical factors determining the performance of the device are the length and configuration of the transducing CNT thread. As described previously, a longer length of CNT thread interlaced through the CNT-PDMS strip provides a larger magnitude of response, pointing to a direct correlation between the response and interaction length. Using a shorter CNT thread invariably results in configurational changes, resulting in inability to decouple the two effects. However, experiments conducted with shorter CNT thread lengths (50\% shorter) and close-spaced configuration exhibit a lower value of resistance (83\% lower) (see Figure S9b,c, Supporting Information) in the normal configuration. Furthermore, the response to biomechanical movement from such a device is also smaller. Given that the electrical resistance of the CNT thread depends only on its length and cross section (ohmic) and is invariant to mechanical movements, the response of the device is also observed to follow this behavior (see Figure S3b, Supporting Information). Furthermore, this also confirms that the CNT thread by itself does not play a direct role in the biomechanical 
Table 1. Comparison of the Active Material, Properties, and Fraction of Active Material for All Relevant Movement-Capture Devices from the Literature

\begin{tabular}{|c|c|c|c|}
\hline material & attributes (advantages and disadvantages) & fraction of active material & refs \\
\hline $\begin{array}{l}\text { MWCNTs }{ }^{a} \text { with Ag paste and indium tin oxide } \\
\text { contacts }\end{array}$ & $\begin{array}{l}\text { pressure sensing in } 0-0.12 \mathrm{MPa} \text { range } \\
\text { rigid, not flexible, and difficult to integrate on clothing }\end{array}$ & $\begin{array}{l}0.5-3 \text { wt \% MWCNTs; } 0.5-3 \text { wt \% } \\
\text { P3HT }\end{array}$ & 7 \\
\hline $\begin{array}{l}\text { thin films of aligned SWCNTs }{ }^{c} \text { on a PDMS } \\
\text { platform }\end{array}$ & $\begin{array}{l}280 \% \text { strain and } 14 \mathrm{~ms} \text { response time } \\
\text { metal electrode components such as } \mathrm{Ti} \text { and } \mathrm{Au} \text { thin films } \\
\text { sensitive to humidity and requires packaging }\end{array}$ & & 12 \\
\hline MWCNTs with chewing gum & $\begin{array}{l}200 \% \text { strain with good sensitivity } \\
\text { sensitive to humidity and requires packaging } \\
\mathrm{Cu} \text { electrodes }\end{array}$ & $2-8$ wt $\%$ MWCNTs & 13 \\
\hline sheets of MWCNTs with a urethane elastomer & $\begin{array}{l}200 \% \text { strain with } 15 \mathrm{~ms} \text { response time } \\
\text { stretchable wire from } \mathrm{Ag} \text { conductive paste as electrodes } \\
\text { effect of humidity and environment is not known }\end{array}$ & & 14 \\
\hline $\mathrm{CB}^{d}$-polyurethane sponges & $\begin{array}{l}\text { wide dynamic pressure-sensing regime } \\
\text { metal contacts restrict human-interfacing }\end{array}$ & 5 wt $\%$ CB & 16 \\
\hline $\mathrm{CNT}-\mathrm{AgNWs}{ }^{e}-\mathrm{PDMS}$ composite & $\begin{array}{l}\text { motion sensor with excellent environmental stability } \\
\text { metal contacts }\end{array}$ & $\sim 50$ wt $\%$ CNTs; $10-50$ wt $\%$ AgNWs & 22 \\
\hline $\begin{array}{l}\text { CNT helices in a thermoplastic elastomer } \\
\text { matrix }\end{array}$ & $\begin{array}{l}\text { wide strain with low hysteresis } \\
\text { Ag paste contacts with bronze electrical leads }\end{array}$ & & 24 \\
\hline AgNWs in PDMS & $\begin{array}{l}\text { stretchable by } 70 \% \text { with response time of } \sim 200 \mathrm{~ms} \\
\text { hysteresis observed } \\
\mathrm{Cu} \text { wires as electrodes }\end{array}$ & $6 \mathrm{mg} / \mathrm{mL} \mathrm{AgNW}$ solution & 27 \\
\hline CB- and CNT-loaded PDMS & $\begin{array}{l}\text { conductive PDMS pads for contacts } \\
\text { strains of } 11.2 \text { and } 22.6 \%\end{array}$ & 25 wt $\%$ CB; 15 wt $\%$ MWCNTs & 35 \\
\hline AgNWs-polyurethane-urea composite & $\begin{array}{l}\sim 50 \% \text { stretching releasing } \\
\text { hysteresis observed } \\
\text { effect of humidity and environment not shown }\end{array}$ & 0.4 wt $\%$ AgNWs & 36 \\
\hline graphene nanoplatelets & $\begin{array}{l}\text { stretchable up to } 150 \% \\
\text { PDMS used as packaging material } \\
\mathrm{Cu} \text { tape and } \mathrm{Ag} \text { paste form the contacts }\end{array}$ & 0.1 wt $\%$ graphene nanoplatelets & 37 \\
\hline MWCNTs and latex nanocomposite & $\begin{array}{l}\text { wide working range } \\
\text { sensor performance dependent on ambient temperature } \\
\text { conductive thread and steel snaps form contacts }\end{array}$ & $1-3$ wt $\%$ MWCNTs & 38 \\
\hline SWCNT and PDMS capacitative strain sensor & $\begin{array}{l}\text { stretchable up to } \sim 300 \% \\
\mathrm{Cu} \text { wires connected through } \mathrm{Ag} \text { paste as contact electrodes }\end{array}$ & as-prepared SWCNT films & 39 \\
\hline $\begin{array}{l}\text { SWCNT-PDMS composite with the CNT } \\
\text { thread as a transducer }\end{array}$ & $\begin{array}{l}25 \% \text { strain, response time of } \sim 200 \mathrm{~ms} \text {, } \\
\text { humidity-independent response and }<2 \% \text { hysteresis } \\
\text { metal-free, all-carbon, nonlithographically fabricated } \\
\text { limited stretchability and robustness }\end{array}$ & 0.016 wt $\%$ of CNTs & $\begin{array}{l}\text { this } \\
\text { report }\end{array}$ \\
\hline
\end{tabular}

tracking and reinforces that the movement-capturing mechanism involves the CNTs embedded in the PDMS matrix.

To understand the underlying mechanism of signal generation, we carry out in situ electromechanical measurements by measuring the changes in resistance across the device during its tensile testing (Figure $5 \mathrm{e}$ ). The relative change in the resistance $(\Delta R / R)$ exhibits a bimodal behavior with extension (Figure 5f). The first part consists of a linear change up to an extension of $7 \mathrm{~mm}$ ( $\sim 26 \%$ strain). Subsequently, a saturationlike behavior up to an extension of $12 \mathrm{~mm}$ is observed, beyond which the device fails ( $40 \%$ strain). Any tensile testing is accompanied by the generation of a simultaneous compressive stress in the transverse direction. Poisson's ratio indicates the extent of generation of such compressive stress (Figure $5 \mathrm{~g}$ ). We observed a similar constriction of the device during the tensile stretching, owing to the positive Poisson ratio of the PDMS matrix (0.5 for PDMS). Driven by this compressive stress, the three dimensionally networked, randomly oriented CNTs embedded in the PDMS matrix reorient and reconfigure (see Figure S6, Supporting Information). Such an effect is observed to produce aligned CNTs in elastomeric matrices, such as PDMS and $n$-butyl nitrile, at higher elongations. ${ }^{31}$ In our device, because the CNTs are present at concentrations very close to the percolation threshold, such reconfigurations of the CNT networks are manifested as changes in the resistance across the CNT-PDMS strip. The CNT thread traversing through the CNT-PDMS strip captures and transduces such changes in electrical resistance that are consequently observed as signals during the experiments. Because the reconfiguration of CNT networks is directly proportional to the compressive stress generated, the response in this regime is expected to exhibit a linear dependence, as is seen in the first part of the response curve (Figure 5f). Compressive stress beyond this regime ( $\sim 26 \%$ strain $)$ does not result in any appreciable change in the CNT network, leading to saturation of the signal generated, as seen in the latter part of the response curve (Figure 5f). This bimodal behavior is exactly reproduced in our in situ experiments as noted above, confirming the mechanism. Such a mechanism operating in this device is thus a symbiotic combination of (a) high Poisson's ratio, resulting in changes to 
the electrical pathway in the device, and (b) the dedicated and precise transduction of these signals by the CNT thread, operating simultaneously during the biomechanical movement. Finally, the saturation domain pertains to the region where the CNT thread embedded in the composite is likely to feel the effect of the tensile stress and therefore does not produce an appreciable response. Any subsequent stretching (>40\% strain) results in either the mechanical failure of the device or the electrical breakdown of the percolation pathway, both of which are irreversible in nature. The proposed mechanism is further confirmed by control experiments carried out with a pure CNT thread, where no such changes are observed.

Strain sensors based on thin films of aligned CNTs operate based on buckling of the film parallel to the strain axis and are operationally similar to the structural deformation of openmesh geometries. Although operational under high strain ${ }^{12}$ (280\%), these devices are extremely difficult to fabricate, require careful packaging to remove ambient effects, and demand extensive electrode fabrication for contacting. In contrast, the device reported here performs under varied humidity conditions without the need for any additional packaging or electrode fabrication and is thus implementable directly on wearable platforms. This has been demonstrated through measurements of our device on platforms ranging from human skin and kinesiologic tapes to robotic arm and NBR gloves. Other reported CNT-based strain sensors largely operate on their preferential alignment during strain. We note that the device in our case is also driven by Poisson's ratio, although the mechanism of capturing the reconfiguration of the CNT network is completely unique. The CNT thread embedded in the device is able to precisely capture minor reconfigurations of the CNT network due to mechanical deformation. Furthermore, our device operates at significantly lower loading of CNTs (0.016 wt \%) compared to that in any other previous report, ${ }^{32-35}$ to the best of our knowledge. This is also responsible for low creep and fast response observed in our device. Furthermore, the final device does not exhibit any sensitivity to the ambient conditions, such as temperature and humidity levels, in contrast to that in other reports. ${ }^{12-14}$ In addition, the integration of the CNT thread onto the elastomeric platform provides a facile method for the measurement of signal and easy interfacing with a variety of surfaces, such as skin, fabric, robot, and gloves.

A comprehensive comparison of the performance and attributes of our device with those reported in the literature is given in Table 1. Although nanocarbons form the predominant choice of the active material, metals and their composites remain the only choice of the electrodes and contacts. $^{7,12-14,16,22,24,35,37-39}$ Furthermore, considering that most of these devices target wearable applications, there are substantially few investigations on the effect of ambient humidity and temperature on the performance of these

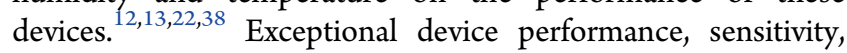
and response time have been achieved through a variety of lithographic and nonlithographic procedures, with the weight fraction of the active material ranging from 0.5 to $50 \mathrm{wt} \%$. In contrast, this report presents the first metal-free, all-carbon device exhibiting fast response time, humidity-invariant response, and excellent reproducibility for $\sim 25 \%$ strain along with excellent versatility for integration into arbitrary platforms. The stretchability and mechanical robustness of our device require improvement, which is being currently pursued through engineering the PDMS curing conditions and the optimization of dispersion parameters of CNTs.

\section{CONCLUSIONS}

In summary, we demonstrate a versatile movement-capturing device employing CNTs as both the functional and transducing components that has been seamlessly integrated onto a variety of ubiquitous platforms. The response from the biomechanical tracking device has been shown to be highly specific to the site of movement and the nature of gesturing. Furthermore, the device enables real-time, continuous, quantitative tracking of movement with signals being directly corroborative of the extent of movement occurring at each musculoskeletal joint. In addition, we have demonstrated an environment-independent response from the device, enabling its direct integration for various applications ranging from physiotherapeutic recuperation and wearable technologies to robotics and defence, where real-time feedback on the type and extent of movement is essential.

\section{EXPERIMENTAL SECTION}

All chemicals and materials were purchased from Sigma-Aldrich and were used directly without any purification, unless mentioned otherwise.

4.1. Synthesis and Dispersion of CNTs and CNT Thread. CNTs were synthesized through the water-assisted chemical vapor deposition ${ }^{18}$ technique with high purity ( $99 \%$, specific surface area $\sim 1000 \mathrm{~m}^{2} / \mathrm{g}$ ), uniformity (length $\sim 350$ $\mu \mathrm{m}$, diameter $\sim 2 \mathrm{~nm})$, and high-aspect ratio $\left(>10^{5}\right)$. The CNTs were dispersed by probe sonication $\left(31 \%\right.$ power at $30{ }^{\circ} \mathrm{C}$ temperature using PKS-750FM; PCI Analytics Pvt. Ltd.) in 4methyl-2-pentanone (MIBK). Immobilization of CNTs on a commercial multiply synthetic yarn was carried out by dipcoating from aqueous, conductive CNT-ink (0.1 wt \%), prepared using sodium deoxycholate as a stabilizing agent. The yarn was subsequently dried $\left(100{ }^{\circ} \mathrm{C}, 30 \mathrm{~min}\right)$ and washed with ethanol to remove the stabilizing agent to result in a CNTimmobilized conductive synthetic yarn (CNT thread). The conductivity of the CNT thread was estimated from two-probe measurements. Iodine doping of as-prepared SWCNTs was carried out by placing SWCNTs in an iodine vapor atmosphere at $100{ }^{\circ} \mathrm{C}$ for $12 \mathrm{~h}$.

4.2. Fabrication of CNT-PDMS Device. The CNTMIBK dispersion was added to a preweighed base elastomer (Sylgard 184; Dow Corning) and mixed thoroughly to ensure uniformity and dispersion. The resulting CNT-MIBKprepolymer slurry was mixed with the curing agent (10:1 ratio). The resulting dispersion was mixed thoroughly and carefully poured into the premade molds for curing. Curing was carried out following the same protocol as that for pure PDMS to yield free-standing CNT-PDMS elastomeric strips.

4.3. Device Fabrication. The CNT-PDMS strips were fabricated with varying compositions of CNTs in PDMS. On the basis of percolation theory, the composite with the highest loading (0.016 wt \%) of CNTs in PDMS was chosen to be the device. The CNT thread was interlaced through the CNTPDMS strips, thereby establishing a contact between the CNTs immobilized on the thread and the CNTs embedded in the elastomer. Thus, the CNT threads can be visualized as metallic wires with significantly higher flexibility and elasticity. Therefore, our approach involved utilizing such conductive strands to 
form serpentine pathways in the PDMS elastomeric matrix, similar to metallic/semiconductor pathways in strain gauges.

4.4. Material Characterization and Mechanical Testing. Micro-Raman spectroscopy was carried out using a WiTeC micro-Raman spectrometer equipped with a neodymium-doped yttrium aluminum garnet $532 \mathrm{~nm}$ excitation laser source. Peak intensities were integrated over $1580 \mathrm{~cm}^{-1}$ (tangential G-band of CNTs) and $2940 \mathrm{~cm}^{-1}$ (asymmetric stretching mode of $\mathrm{C}-\mathrm{H}$ ) to elucidate the spatial distribution of CNTs and PDMS, respectively. Confocal single-point depth profiling was carried out over a depth of $10 \mu \mathrm{m}$ to ascertain the distribution of CNTs in the PDMS matrix. X-ray photoelectron spectroscopy was carried out using AXIS Supra, Kratos Analytical, Shimadzu, employing an $\mathrm{Al} \mathrm{K} \alpha$ source. Absorption spectrophotometry was carried out using UV-3600, Shimadzu, in the transmission mode with a pure PDMS sample as the reference. Confocal laser scanning microscopic imaging in transmission mode was carried out using a FV500 TIEMPO FLUOVIEW microscope. Optical images through a sample thickness of $200 \mu \mathrm{m}$ were obtained in a confocal fashion from top to bottom at sequentially decreasing focal planes. Mechanical testing was conducted using an Instron 3366 universal tensile machine (American society for testing and materials (ASTM) D3039), with a capacity of $10 \mathrm{kN}$ and at a strain change of $0.1 \mathrm{~mm} / \mathrm{min}$. Secondary electron microscopy was carried out using FEI Quanta 200 SEM. Four-dimensional $\mathrm{X}$-ray imaging was carried out on a $3 \mathrm{D}$ volume element, using Xradia Versa 520, Zeiss. Tensile and bending modes were performed using a custom-built tensile tester and bending tester-jig that were designed according to the requirements posed by the composites and confined to ASTM D3039 standards. The tensile tester consisted of a fixed clamp and a movable clamp, held in the same plane, onto which the sample was fixed. The movable clamp was used for repeated tensile testing of the sample. The entire assembly was fabricated in SS 301 grade steel. The SD for such repeated testing was calculated through established data analytical methods, given by

$$
\mathrm{SD}=\sqrt{\frac{\sum\left(X-X_{\mathrm{m}}\right)^{2}}{n}}
$$

where $X$ represents individual data points, $X_{\mathrm{m}}$ represents their mean, and $n$ is the total number of data points.

4.5. Electrical Measurements. KEITHLEY 2110 digital multimeter (DMM), interfaced to computer, was used for electrical measurements. Two-probe resistance of the CNT thread during various stages of its fabrication was measured using a Bio-Logic SP-300 electrochemical workstation. The flexibility and mechanical robustness of the CNT thread enabled treating it exactly like a normal thread. Thus, either end of CNT threads was twisted across a battery and an lightemitting diode (LED), respectively, to complete the circuit, resulting in glowing of the LED. Both the current-voltage measurement and the demonstration were absolutely invariant for all possible configurational changes of the CNT thread, such as bending, twisting, stretching, and winding.

4.6. Real-Time Movement Capturing. The device was conformally attached to various substrates at arbitrary sites of measurement using a medical adhesive (to prevent slippage). Accordingly, the performance of the device was evaluated by directly interfacing on human skin. Biomechanical movements were also tracked by attaching the device on NBR gloves and kinesiologic tapes that were worn on a human hand.
Biomechanical movements at various joints on the human hand, such as the proximal IP joint (finger), MP joint (knuckle), and CM joint (wrist), were monitored. Hand movements were artificially simulated using a commercially procured robotic arm (Robotic Hand, KidzLabs). The signals in all of these cases were monitored with the help of a KEITHLEY $2110 \mathrm{DMM}$, at time intervals of $50 \mathrm{~ms}$ interfaced with a computer for automated data acquisition. The two ends of the CNT thread formed the electrodes for all such measurements. Measurements were continuously acquired while bending the joints to various extents.

\section{ASSOCIATED CONTENT}

\section{Supporting Information}

The Supporting Information is available free of charge on the ACS Publications website at DOI: 10.1021/acsomega.7b00491.

Absorption spectrophotometry results of the CNTPDMS strip, secondary electron microscopy images of the pure thread and CNT thread, transmission electron microcopy image of the CNT thread, conductivity studies on the CNT thread, site-specific device response, $\mathrm{X}$-ray photoelectron spectroscopy of iodine-doped CNTs, optical images of the CNTs distributed within the CNT-PDMS matrix, confocal micro-Raman depth mapping, confocal laser scanning microscopy, distribution of CNTs in the matrix, mechanical properties and cyclability of the device (Figures S1-S9); image analysis and quantification of CNT distribution in the device (Table S1) (PDF)

\section{AUTHOR INFORMATION}

\section{Corresponding Author}

*E-mail: csubbu@chem.iitb.ac.in.

\section{ORCID}

Chandramouli Subramaniam: 0000-0001-9901-7184

\section{Author Contributions}

P.R. and C.S. conceptualized and performed the experiments, co-wrote the manuscript, and analyzed the data. P.R. and M.K.J. were involved in the characterization of the CNT thread. K.H. helped in the discussion of data.

\section{Notes}

The authors declare no competing financial interest.

\section{ACKNOWLEDGMENTS}

P.R and C.S. acknowledge the research support from the Indian Institute of Technology Bombay (IIT-B) and Industrial Research and Consultancy Centre (IRCC) and also acknowledge Shanideo N. Jadhav and Strength of Materials Lab, Mechanical Department, IIT-Bombay, for mechanical measurements.

\section{REFERENCES}

(1) Hammock, M. L.; Chortos, A.; Tee, B. C.-K.; Tok, J. B.-H.; Bao, Z. 25th Anniversary Article: The Evolution of Electronic Skin (ESkin): A Brief History, Design Considerations, and Recent Progress. Adv. Mater. 2013, 25, 5997-6038.

(2) Dahiya, R. S.; Metta, G.; Valle, M.; Sandini, G. Tactile Sensing from Humans to Humanoids. IEEE Trans. Rob. 2010, 26, 1.

(3) Kuribara, K.; Wang, H.; Uchiyama, N.; Fukuda, K.; Yokota, T.; Zschieschang, U.; Jaye, C.; Fischer, D.; Klauk, H.; Yamamoto, T.; Takimiya, K.; Ikeda, M.; Kuwabara, H.; Sekitani, T.; Loo, Y.-L.; 
Someya, T. Organic Transistors with High Thermal Stability for Medical Applications. Nat. Commun. 2012, 3, No. 723.

(4) Ghodssi, R.; Lin, P. MEMS Materials and Processes Handbook, 1st ed.; Springer: New York, 2011; pp 273-344.

(5) Lau, S. L.; David, K. In Movement Recognition Using the Accelerometer in Smartphones, Presented at Future Network and Mobile Summit, Florence, June 16-18, 2010; p 1.

(6) Rome, K.; Cowieson, F. A Reliability Study of the Universal Goniometer, Fluid Goniometer, and Electrogoniometer for the Measurement of Ankle Dorsiflexion. Foot Ankle Int. 1996, 17, 28-32.

(7) Hwang, J.; Jang, J.; Hong, K.; Kim, K. N.; Han, J. H.; Shin, K.; Park, C. E. Poly(3-hexylthiophene) wrapped Carbon Nanotube/Poly (dimethylsiloxane) Composites for use in Finger-Sensing Piezoresistive Pressure Sensors. Carbon 2011, 49, 106-110.

(8) Sekitani, T.; Noguchi, Y.; Hata, K.; Fukushima, T.; Aida, T.; Someya, T. A Rubberlike Stretchable Active Matrix using Elastic Conductors. Science 2008, 321, 1468-1472.

(9) Sekitani, T.; Nakajima, H.; Maeda, H.; Fukushima, T.; Aida, T.; Hata, K.; Someya, T. Stretchable Active-Matrix Organic Light-Emitting Diode Display using Printable Elastic Conductors. Nat. Mater. 2009, 8, 494-499.

(10) Someya, T.; Sekitani, T.; Iba, S.; Kato, Y.; Kawaguchi, H.; Sakurai, T. A Large-Area, Flexible Pressure Sensor Matrix with Organic Field-Effect Transistors for Artificial Skin Applications. Proc. Natl. Acad. Sci. U.S.A. 2004, 101, 9966-9970.

(11) Duong, S.; Choi, M.-H. Interactive Full-Body Motion Capture using Infrared Sensor Network. Int. J. Comput. Graphics Anim. 2013, 3, 41.

(12) Yamada, T.; Hayamizu, Y.; Yamamoto, Y.; Yomogida, Y.; IzadiNajafabadi, A.; Futaba, D. N.; Hata, K. A Stretchable Carbon Nanotube Strain Sensor for Human-Motion Detection. Nat. Nanotechnol. 2011, 6, 296-301.

(13) Darabi, M. A.; Khosrozadeh, A.; Wang, Q.; Xing, M. Gum Sensor: A Stretchable, Wearable, and Foldable Sensor based on Carbon Nanotube/Chewing Gum Membrane. ACS Appl. Mater. Interfaces 2015, 7, 26195-26205.

(14) Suzuki, K.; Yataka, K.; Okumiya, Y.; Sakakibara, S.; Sako, K.; Mimura, H.; Inoue, Y. Rapid-Response, Widely Stretchable Sensor of Aligned MWCNT/Elastomer Composites for Human Motion Detection. ACS Sens. 2016, 1, 817-825.

(15) Li, Y.; Mai, C.-K.; Phan, H.; Liu, X.; Nguyen, T.-Q.; Bazan, G. C.; Chan-Park, M. B. Electronic Properties of Conjugated Polyelectrolyte/Single-Walled Carbon Nanotube Composites. Adv. Mater. 2014, 26, 4697-4703.

(16) Wu, X.; Han, Y.; Zhang, X.; Zhou, Z.; Lu, C. Large-Area Compliant, Low-Cost, and Versatile Pressure-Sensing Platform Based on Microcrack-Designed Carbon Black@Polyurethane Sponge for Human-Machine Interfacing. Adv. Funct. Mater. 2016, 26, 62466256.

(17) Wu, X.; Han, Y.; Zhang, X.; Lu, C. Highly Sensitive, Stretchable, and Wash-Durable Strain Sensor Based on Ultrathin Conductive Layer@Polyurethane Yarn for Tiny Motion Monitoring. ACS Appl. Mater. Interfaces 2016, 8, 9936-9945.

(18) Hata, K.; Futaba, D. N.; Mizuno, K.; Namai, T.; Yumura, M.; Iijima, S. Water Assisted Highly-Efficient Synthesis of Impurity-Free Single Walled Carbon Nanotubes. Science 2004, 306, 1362-1364.

(19) Ata, S.; Mizuno, T.; Nishizawa, A.; Subramaniam, C.; Futaba, D. N.; Hata, K. Influence of Matching Solubility Parameter of Polymer Matrix and CNT on Electrical Conductivity of CNT/Rubber Composite. Sci. Rep. 2014, 4, No. 7232.

(20) Subramaniam, C.; Yasuda, Y.; Takeya, S.; Ata, S.; Nishizawa, S.; Futaba, D. N.; Yamada, T.; Hata, K. Carbon Nanotube-Copper Exhibiting Metal-like Thermal Conductivity and Silicon-like Thermal Expansion for Efficient Cooling of Electronics. Nanoscale 2014, 6, 2669-2674.

(21) Subramaniam, C.; Yamada, T.; Kobashi, K.; Sekiguchi, A.; Futaba, D. N.; Yumura, M.; Hata, K. One Hundred fold Increase in Current Carrying Capacity in a Carbon Nanotube-Copper Composite. Nat. Commun. 2013, 4, No. 2202.
(22) Jeon, J.-Y.; Ha, T.-J. Waterproof Electronic-Bandage with Tunable Sensitivity for Wearable Strain Sensors. ACS Appl. Mater. Interfaces 2016, 8, 2866-2871.

(23) Mizuno, K.; Ishii, J.; Kishida, H.; Hayamizu, Y.; Yasuda, S.; Futaba, D. N.; Yumura, M.; Hata, K. A Black-Body Absorber from Vertically-Aligned Single Walled Carbon Nanotubes. Proc. Natl. Acad. Sci. U. S. A. 2009, 106, 6044.

(24) Li, C.; Cui, Y.-L.; Tian, G.-L.; Shu, Y.; Wang, X.-F.; Tian, H.; Yang, Y.; Wei, F.; Rena, T.-L. Flexible CNT-Array Double Helices Strain Sensor with High Stretchability for Motion Capture. Sci. Rep. 2015, 5, No. 15554.

(25) Hareem, M.; Bockrath, M. Elastomeric Carbon Nanotube Circuits for Local Strain Sensing. Appl. Phys. Lett. 2006, 89, No. 173131.

(26) Mole, R. H. The Relative Humidity of the Skin. J. Physiol. 1948, 107, 399-411.

(27) Amjadi, M.; Pichitpajongkit, A.; Lee, S.; Ryu, S.; Park, I. Highly Stretchable and Sensitive Strain Sensor based on Silver NanowireElastomer Nanocomposite. ACS Nano 2014, 8, 5154.

(28) Zhao, Y.; Wei, J.; Vajtai, R.; Ajayan, P. M.; Barrera, E. V. Iodine Doped Carbon Nanotube Cables exceeding Specific Electrical Conductivity of Metals. Sci. Rep. 2011, 1, No. 83.

(29) Subramaniam, C.; Sekiguchi, A.; Yamada, T.; Futaba, D. N.; Hata, K. Nano-Scale, Planar and Multi-Tiered Current Pathways from a Carbon Nanotube-Copper Composite with High Conductivity, Ampacity and Stability. Nanoscale 2016, 8, 3888-3894.

(30) Song, Y. S.; Youn, J. R. Influence of Dispersion States of Carbon Nanotubes on Physical Properties of Epoxy Nanocomposites. Carbon 2005, 43, 1378-1385.

(31) Mensah, B.; Kim, H. G.; Lee, J.-H.; Arepalli, S.; Nah, C. Carbon Nanotube-Reinforced Elastomeric Nanocomposites: A Review. Int. J. Smart Nano Mater. 2015, 6, 211-238.

(32) Slobodian, P.; Riha, P.; Benlikaya, R.; Svoboda, P.; Petras, D. A Flexible Multifunctional Sensor based on Carbon Nanotube/Polyurethane Composite. IEEE Sens. J. 2013, 13, 4045-4048.

(33) Toprakci, H. A. K.; Kalanadhabhatla, S. K.; Spontak, R. J.; Ghosh, T. K. Polymer Nanocomposites containing Carbon Nanofibers as Soft Printable Sensors exhibiting Strain-Reversible Piezoresistivity. Adv. Funct. Mater. 2013, 23, 5536-5542.

(34) Yan, C.; Wang, J.; Kang, W.; Cui, M.; Wang, X.; Foo, C. Y.; Chee, K. J.; Lee, P. S. Highly Stretchable Piezoresistive GrapheneNanocellulose Nanopaper for Strain Sensors. Adv. Mater. 2014, 26, 2022-2027.

(35) Lu, N.; Lu, C.; Yang, S.; Rogers, J. Highly Sensitive SkinMountable Strain Gauges based Entirely on Elastomers. Adv. Funct. Mater. 2012, 22, 4044-4050.

(36) Kim, D.-H.; Yu, K.-C.; Kim, Y.; Kim, J.-W. Highly Stretchable and Mechanically Stable Transparent Electrode Based on Composite of Silver Nanowires and Polyurethane-Urea. ACS Appl. Mater. Interfaces 2015, 7, 15214-15222.

(37) Park, J. J.; Hyun, W. J.; Mun, S. C.; Park, Y. T.; Park, O. O. Highly Stretchable and Wearable Graphene Strain Sensors with Controllable Sensitivity for Human Motion Monitoring. ACS Appl. Mater. Interfaces 2015, 7, 6317-6324.

(38) Wang, L.; Loh, K. J. Wearable carbon nanotube-based fabric sensors for monitoring human physiological performance. Smart Mater. Struct. 2017, 26, No. 055018.

(39) Cai, L.; Song, L.; Luan, P.; Zhang, Q.; Zhang, N.; Gao, Q.; Zhao, D.; Zhang, X.; Tu, M.; Yang, F.; Zhou, W.; Fan, Q.; Luo, J.; Zhou, W.; Ajayan, P. M.; Xie, S. Super-stretchable, Transparent Carbon Nanotube-Based Capacitive Strain Sensors for Human Motion Detection. Sci. Rep. 2013, 3, No. 3048. 